\title{
Concentración de plaguicidas en agua, sedimentos y ostiones (Crassostrea rizophorae) de la Laguna de Bluefields, RAAS, Nicaragua
}

\section{'Billy Francis Ebanks Mongalo; ',2 Juan Suárez Sánchez; ' Eduardo Siu Estrada; 'Juan José Montoya Arguello; 'Daniel Álvaro Mairena Valdivia; 'Asdrubal Flores Pacheco; ${ }^{3}$ Van der Wal, Johannes Cornelis; ${ }^{2}$ Pedro Valencia Quintana.'}

La laguna de Bluefields es el receptor natural de los escurrimientos de agua de lluvia de las cuencas de los ríos Escondido (61) y Kukra River (63) (Umañas, 2002). En la cuenca de río Escondido se han desarrollado actividades agropecuarias intensivas, con el cultivo del banano (Musa sp.) desde la década de 1960; con el cultivo de la caña de azúcar (Zacharum officinarum) durante las décadas de 1970 y 1980, y actualmente, con el cultivo de la palma africana (Elais guiniensis).

En la cuenca de Kukra River, la actividad agropecuaria inicia, en la década de 1990, con el cultivo del coco (Cocos nucifera), granos básicos y hortalizas. Para estas actividades se han utilizado y utilizan grandes cantidades de plaguicidas para el control de malezas e insectos, en muchas ocasiones

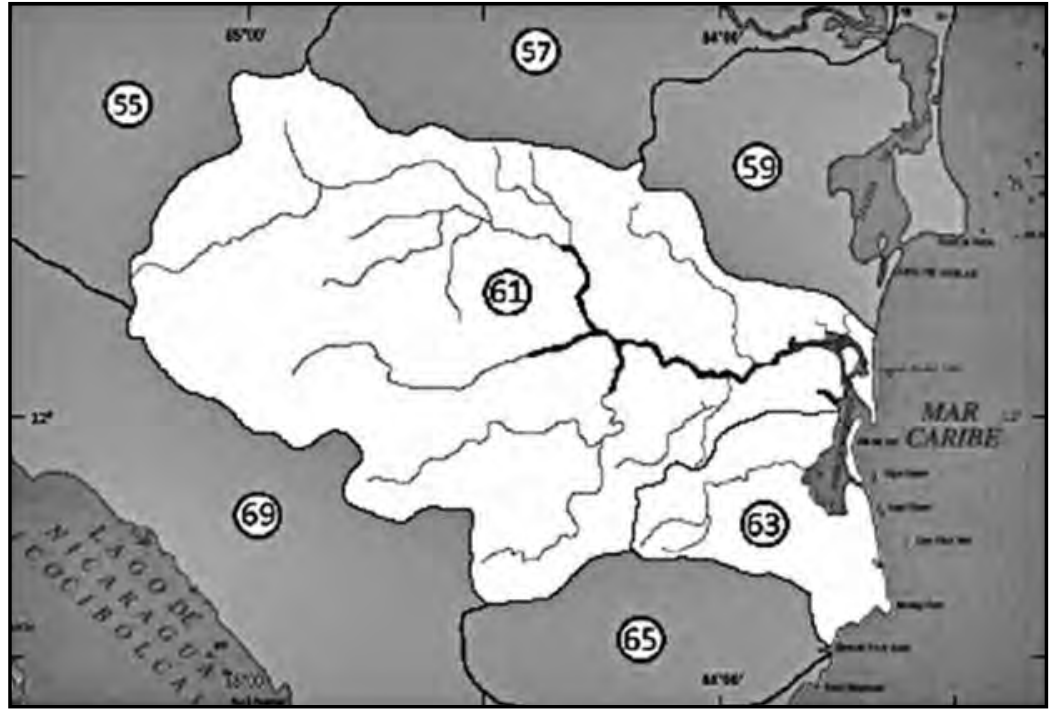

Figura 1. Laguna de Bluefields y sus cuencas contribuyentes (61 y 63). Región Autónoma del Atlántico Sur, Nicaragua (modificada de INETER, 2012). sin el menor cuidado en su manejo (Espinosa, 2005 y REPCAR, 2012). Con las constantes lluvias que suceden en las cuencas, en promedio 2,722 mm para la cuenca río Escondido y 3,710 mm para la cuenca Kukra River, los plaguicidas son transportados por los escurrimientos de agua hacia el sistema de drenaje (Varca, 2012), por lo que se espera que estén presentes en la laguna de Bluefields.

Los cultivos intensivos son los que más utilizan plaguicidas; el cultivo del maíz (Zea mays) es uno de los que más aplica (Blanchound et al., 2004, 2007; Wittmer et al., 2010). Los insecticidas y fungicidas son los más empleados en la agricultura intensiva representando el $12 \%$ del total (Blanchound et al., 2004). El uso de plaguicidas en el mundo va en incremento, así, por ejemplo, en los últimos

Facultad de Recursos Naturales y Medio Ambiente. Bluefields Indian and Caribbean University. Bluefields, Región Autónoma del Atlántico Sur, Nicaragua. ciabbicu@gmail.com.

2 Laboratorio de Medio Ambiente. Facultad de Agrobiología. Universidad Autónoma de Tlaxcala. Ixtacuixtla, Tlaxcala, México. jsuarezs71@gmail.com.

Agroecología, El Colegio de la Frontera Sur. Villahermosa, México. 
años, en Filipinas han incrementado en un $28 \%$ en la producción de vegetales y tubérculos, para la protección contra insectos e infecciones fúngicas y del total que se usa, el 37\% es para la producción de tubérculos (Varca, 2012). Actualmente, en la Región Autónoma del Atlántico Sur de Nicaragua, los plaguicidas más utilizados son el Gramoxoneen un 41\%; glifosato, cypermetrina y 2-4D con un $17 \%$ cada uno; otros de menor frecuencia de uso en un $8 \%$.

En general, la agricultura intensiva combinada con escurrimientos superficiales, lixivia y envía el agua de lluvia a los canales de drenaje, provocando un incremento de los niveles de nutrientes y plaguicidas en los ríos. Los factores que afectan la contaminación del agua superficial son la cercanía a las áreas de producción (rivera de los ríos), características del cuerpo de agua (área, profundidad y flujo), características de los campos circundantes (tipo de suelo, cubierta vegetal, pendiente y distancia a los cuerpos de agua) y condiciones atmosféricas (temperatura, humedad, viento y precipitación) (Bach et al., 2001 y Vryzas et al., 2009).

El aporte medio de plaguicidas a los cuerpos de agua varía considerablemente entre regiones y depende altamente de las tasas de aplicación, características químicas de los plaguicidas y las condiciones naturales durante su aplicación (Huber et al., 2000). La movilidad de los plaguicidas en el suelo y desde ahí su biodisponibilidad y transferencia a otro compartimiento ambiental, depende de una variedad de complejos procesos de dinámica física, química y biológica, incluyendo absorción-desabsorción, volatilización, degradación química y biológica, absorción por plantas, escurrimiento y lixiviación (Vryzas et al., 2009). La concentración de pesticidas tiende a ser mayor en pequeñas cuencas con intensa agricultura y cuando se usa equipo de aspersión defectuosos (Holvoet et al., 2007).

Los plaguicidas aplicados al suelo son los más frecuentemente detectados. Altas concentraciones de plaguicidas son detectadas dentro de los dos meses después de su aplicación. Concentraciones extremas de plaguicidas son detectadas al principio de la estación de irrigación y justo después de eventos de lluvia fuertes (Vryzas et al., 2009). Desde que los plaguicidas ingresan a los ríos están expuestos a diferentes procesos físicos, químicos y microbiológicos, que determinan su destino. En cuencas grandes, menos del $2 \%$ de la masa de los plaguicidas aplicados finalmente se pierden hacia las aguas superficiales y ocurren principalmente durante y justo después del periodo de aplicación. Así también, los factores cruciales que influyen sobre la pérdida de plaguicidas a la cuenca son las condiciones del tiempo, tipo de suelo, uso de la tierra, propiedades intrínsecas de los compuestos y fuentes puntuales. Las concentraciones de plaguicidas tienden a ser más altas en lugares intervenidos por el hombre con cultivos intensivos y la continua fumigación (Holvoet et al., 2007).

El mal manejo de los plaguicidas afecta a los ecosistemas y a las poblaciones humanas que habitan en ellos, ya que sus organismos son expuestos directa o indirectamente a través de la trama trófica y los ciclos biogeoquímicos (Maloschik, 2007). Los efectos del mal manejo de los plaguicidas se observan a corto plazo (intoxicaciones graves, agudas y crónicas) y a largo plazo (por efectos de bioconcentración, bioacumulación y desarrollo de plagas resistente). Se han descubierto problemas ecotoxicológicos (ejemplo, efecto de descontrol endocrinológico), relacionados con residuos de plaguicidas en el agua para consumo humano (Tejada et al., 1995; Berg, 2001 y Varca, 2012).

Los residuos en los equipos de aspersión, fallas en los equipos (fugas), mala aplicación (dosis elevadas), condiciones ambientales adversas, son sólo algunos ejemplos del mal manejo de los plaguicidas que contribuyen a la contaminación del ambiente (Kreuger, 1998 y Neumann et al., 2002). Para reducir la contaminación del agua por causa de plaguicidas es importante conocer su composición química y tiempo de vida (estabilidad y degradación) (Hubert et al., 2000), así como proporcionar entrenamiento para el manejo adecuado de éstos, en especial a los usuarios y los responsables de su comercialización (Blanchound et al., 2004). La detección de plaguicidas en la Laguna de Bluefields permitirá realizar medidas integrales que coadyuven a prevenir y reducir la contaminación del ecosistema lagunar y las poblaciones humanas que se interrelacionan con él.

El objetivo de este trabajo fue determinar las concentraciones de plaguicidas en agua, sedimento y ostiones (Crassostrea rizophorae) en la laguna de Bluefields, que permitan identificar el riesgo ambiental al que están expuestos el sistema lagunar y las poblaciones humanas que se desarrollan en sus alrededores.

\section{MATERIALES Y MÉTODOS}

\section{Área de Estudio}

La Laguna de Bluefields se encuentra ubicada entre los $11^{\circ} 48^{\prime}$ y los $12^{\circ} 11^{\prime}$ de longitud norte y entre los $83^{\circ} 41^{\prime}$ y $83^{\circ} 50^{\prime}$ de longitud oeste, en la Región Autónoma del 
Atlántico Sur (RAAS), Nicaragua. Tiene una área total de $176 \mathrm{~km}^{2}$, una longitud aproximada de $30.5 \mathrm{~km}$, una anchura promedio de $6 \mathrm{~km}$ y posee una profundidad media de $1 \mathrm{~m}$, las mayores profundidades están en la parte norte. En la laguna se presenta un área de transición entre la cuenca de escurrimiento del río Escondido (cuenca 63, que tiene un área de 11,650 $\mathrm{km}^{2}$ y una precipitación promedio anual de 2,722 mm), la cuenca de escurrimiento del río Kukra (cuenca 61, que tiene un área de 1,593 km² y una precipitación promedio anual de $3710 \mathrm{~mm}$ ) y el mar Caribe (Fig. 1); se une al mar en la parte sur por la Barra de Hone Sound y en la parte norte por la Barra del Puerto el Bluff. La Laguna posee varias islas, que en su mayoría están deshabitadas (Umañas, 2002).

\section{Metodología}

Se determinaron 11 puntos de muestreo distribuidos en las áreas de interés de acuerdo al estudio (Fig. 2). Tres puntos distribuidos en la descarga del río Escondido a la laguna, que es el de mayor aporte de agua dulce, un punto en la barra del Bluff, un punto frente a la ciudad de Bluefields, dos puntos en la parte media, un punto en la parte sur, dos puntos en la desembocadura del río Kukra y uno en la barra de Hone Sound.

En los puntos seis y siete que se ubican en la zona donde habita el ostión (Crassostrea rizophorae), se tomaron muestras de ésta especie. En los puntos dos y nueve se tomaron muestras de agua, y en los puntos restantes ( 1,3 , $4,5,8,10$ y 11) se tomaron muestras de agua y sedimentos.

El agua se obtuvo mediante un muestreo simple a $50 \mathrm{~cm}$ de la superficie. El sedimento se obtuvo con un nucleador de acero inoxidable, con núcleos de cinco cm de diámetro por $11.25 \mathrm{~cm}$ de alto. Para la muestra de ostiones se obtuvieron directamente del banco hasta conseguir 500 gr de tejido de ostión.

Todas las muestras fueron recolectadas en el mes de noviembre de 2011.

El análisis de residuos de plaguicidas fue realizado por el Laboratorio para el Análisis de Contaminantes de la Universidad Nacional Autónoma de Nicaragua, León. El análisis de plaguicidas en agua lo realizaron con el método por partición liquido-liquido y cuantificación por Cromatografía de Gases con Detector Captura de Electrones (ECD) y Detector Termiónico (NPD), según EPA 508. La determinación de plaguicidas en sedimento fue por extracción asistida por ultrasonido y después por el mismo método que el agua, según EPA 8141ª-3550b. La detección de plaguicidas en ostiones fue por homogenización con disolventes orgánicos y después por el mismo método que el agua, según PAM 302-304. Los resultados en todos los casos fueron verificados por medio de Cromatografía de Gases con Detector de Masas.

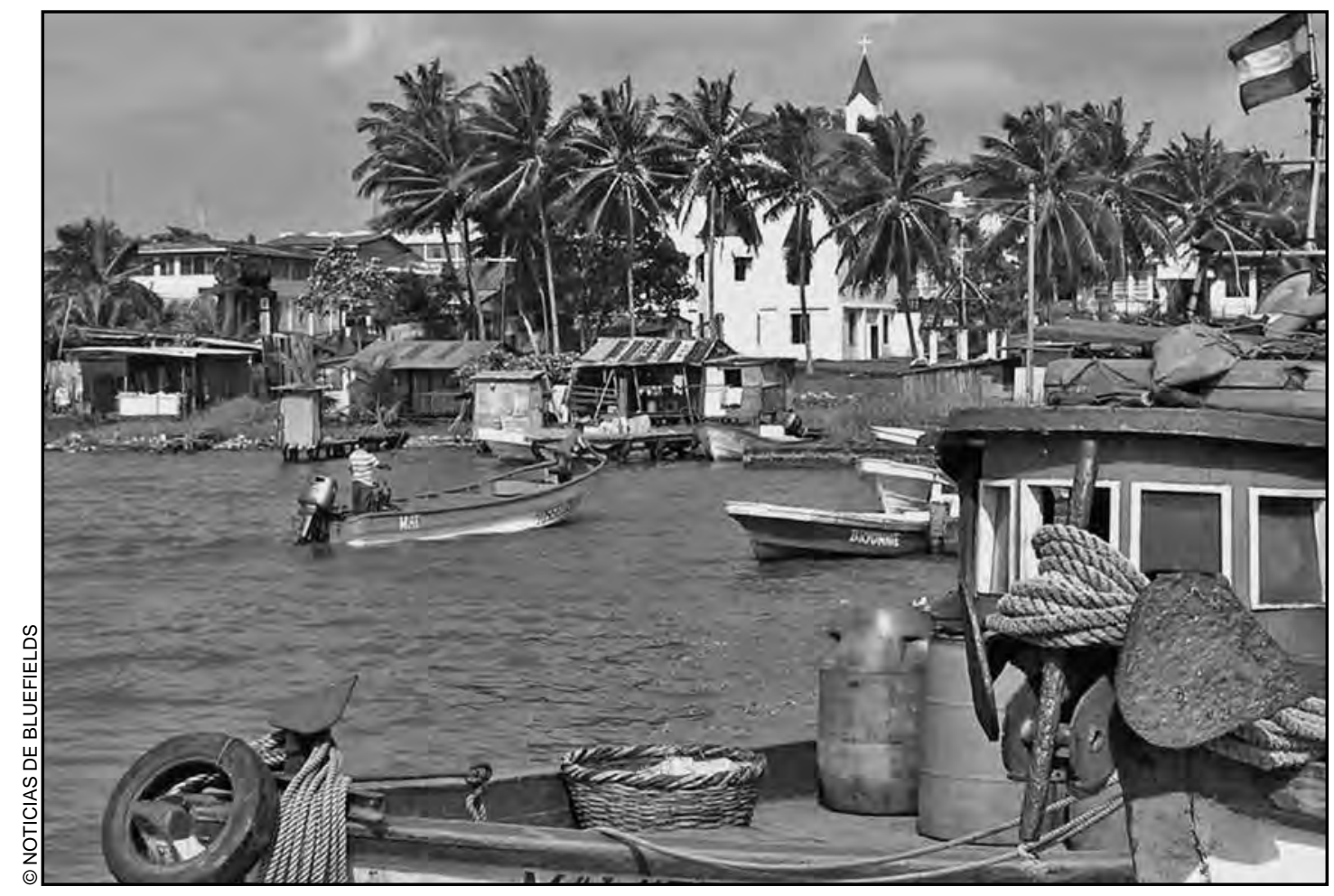

Bahia de Bluefieds. 


\section{RESULTADOS Y DISCUSIÓN}

De los análisis de plaguicidas a las muestras de agua, sedimento y ostiones de la Laguna de Bluefields se observó lo siguiente.

En la muestra de agua del punto cuatro ubicado en la Barra del Bluff, punto de interacción entre la descarga del río Escondido y el mar Caribe, se detectaron los insecticidas organoclorados Dieldrín, PP-DDT y OP-DDD, en concentraciones de 16, 43 y $17 \mu \mathrm{g} \mathrm{L}^{-1}$, respectivamente, y el insecticida-acaricida Terbufos en concentración de 17 $\mathrm{mg} \mathrm{L}^{-1}$. Estas concentraciones de pesticidas sobrepasan los límites estándar europeos de calidad de aguas crudas (2 mg $\mathrm{L}^{-1}$ ) (Botta et al., 2012). Los plaguicidas son transportados durante los eventos de escurrimiento. En Alemania, los escurrimientos de las cuencas son la mayor fuente de contaminación difusa de los ríos por plaguicidas (Holvoet et al., 2007). Consideramos que la presencia de plaguicidas en este punto de muestreo se debe a los aportes que hace el río Escondido, ya que recolecta los escurrimientos de la cuenca 61. Estos plaguicidas detectados fueron usados intensivamente, en la década de 1960, para los cultivos de banano, y en la de 1970 para los cultivos de caña de azúcar; el Terbufos ha sido utilizado para los cultivos de coco (Com. Per.). Este comportamiento ya fue observado en el río San Juan en 2004 (sur de Nicaragua), donde reportaron la presencia de PP-DDE, lindano, PP-DDT y Endosulfano II, en muestras de agua, Dieldrín PP-DDE y lindano en partículas en suspensión (Cuadra y Vammen, 2010).

En los análisis de sedimentos en los puntos 1, 3, 4, 5, 8 y 10, se detectó Clorpirifos (insecticida organofosforado), pero no se pudo cuantificar. Los puntos uno, tres y cuatro, corresponden a la desembocadura del río Escondido, el punto cinco a las descargas de la ciudad de Bluefields, el punto ocho a la desembocadura del Kukra River y el punto diez a la desembocadura del arroyo Docuno, éstos dos últimos correspondientes a la cuenca 63. A excepción del punto cinco, consideramos que la presencia de este insecticida en las muestras de sedimentos se debe a los aportes que hacen los ríos Escondido, Kukra y Docuno, al igual que los plaguicidas encontrados en el punto 4 (Holvoet et al., 2007).

En las dos muestras de ostiones se encontró OP-DDE, en concentraciones $<1.3 \mathrm{mg} \mathrm{Kg}^{-1}$. En el punto seis se identificó además PP-DDE en concentración $<0.36 \mathrm{mg} \mathrm{Kg}^{-1}$. En el punto 7 se detectó Clorpirifos en concentración $<1.1 \mathrm{mg}$ $\mathrm{Kg}^{-1}$. Concentraciones menores a los límites permisibles para consumo humano de acuerdo a las diferentes normas establecidas por las diferentes agencias internacionales (Rueda et al., 1997). Considerando que el ostión es filtrador, la detección de estos plaguicidas, infiere la existencia de ellos en la laguna en menores concentraciones, por lo que no fue posible detectarlos en los otros componentes (agua y sedimentos), a excepción del Clorpirifos.

La identificación de siete plaguicidas en la laguna de Bluefields, cuatro en agua (Dieldrín, PP-DDT, OP-DDD y terbufos), uno en sedimentos (Clorpirifos) y tres en ostiones (OP-DDE, PP-DDE y Clorpirifos), confirma la creciente preocupación por la contaminación de los ríos, lagunas costeras y bancos de pesca del Mar Caribe nicaragüense y la necesidad de generar información sobre el grado de contaminación existente, para coadyuvar a determinar las fuentes y desarrollar estrategias adecuadas dirigidas a reducir el escurrimiento de plaguicidas a esta región (Cuadra y Vammen, 2010).

\section{AGRADECIMIENTOS}

Al programa PASMA-DANIDA por el financiamiento otorgado a esta investigación.

Al Centro de Investigaciones en la Salud, Trabajo y Ambiente (CISTA) de Universidad Nacional Autónoma de Nicaragua - León, por el análisis de residuos de plaguicidas en muestras de agua, sedimento y ostiones provenientes de la bahía de Bluefields, Nicaragua.

Al Programa estratégico Cuerpos Académicos, Calidad y Productividad para su Internacionalización (CACyPIUATx-2013) por su apoyo para esta investigación. 


\section{BIBLIOGRAFÍA}

Bach, M; Huber, A; Frede, HG. 2001. "Input pathways and river load of pesticides in Germany -a national scale modeling assessment. Water Sci. Technol. 43(5):261-268.

Berg, H. 2001. "Pesticide use in rice and rice-fish farms in the Mekong Delta, Vietnam. Crop Protection. 20:897-905. Blanchound, H; Farrugia, F; Mouchel, JM. 2004. "Pesticide uses and transfers in urbanised catchments". Chemosphere. 55:509-913.

Blanchound, H; Moreau, GE; Farrugia, F; Chevreuil, M; Mouchel, JM. 2007. "Contribution by urban and agricultural pesticide uses to water contamination at the scale of the Marne watershed”. Sci. Total Environ. 375:168-179.

Brenes, C. 1999. Caracterización hidrológica de la Laguna de Perlas, Nicaragua. Bluefields, Nicaragua. Pp 46.

Botta, F.; Fauchon, N.; Blanchoud, H.; Chevreuil, M.; Guery, B. (2012). "Phyt’Eaux Citéx: Application and validation of a programme to reduce surface water contamination urban pesticides”. Chemosphere 86: 166-176.

Cuadra, J. y Vammen, K. 2010. "Escurrimiento de Plaguicidas al Mar Caribe Nicaragüense - Establecimiento de un Sistema de Monitoreo Ambiental”. Universidad y Ciencia. No. 8. Año 5. 27-32.

Dumailo, S. 2003. Evaluación de la problemática ambiental por medio del estudio de algunos aspectos de sedimentación y contaminación en la laguna de Blefields, RAAS, Nicaragua. Tesis Master en Ciencias del Agua. Universidad Nacional Autónoma de Nicaragua.

Espinosa, U. H. 2005. Inventario Nacional de Plaguicidas COP en Nicaragua. Gobierno de Nicaragua, Ministerio del Ambiente y los Recursos Naturales. 116 pp.

Holvoet, KMA; Seuntjens, P; Vanrolleghem, PA. 2007. "Monitoring and modeling pesticide fate in surface waters at the catchment scale”. Ecological Modelling. 209:53-64.

Huber A, Bach M, Frede, HG. 2000. "Pollution of surface waters with pesticides in Germany: modeling non-point source inputs”. Agriculture, Ecosystems and Environment. 80:191-204.

INETER. 2012. República de Nicaragua. Cuencas hidrográficas. Instituto Nicaragüense de Estudios Territoriales. En Internet: www.ineter.gob.ni.

Kreuger, J. 1998. "Pesticides in stream water within an agricultural catchment in southern Sweden, 1990-1996”. Sci. Total Environ. 216:227-251.

Maloschik, E; Ernst, A; Hegedüs, G; Darvas, B; Székács, A. 2007. “Monitoring water-polluting pesticides in Hungary”. Microchemical Journal. 85:88-97.

Neumman, M; Schulz, R; Chafer, K; Muller, W. Mannheller, W. Liess, M. 2002. "The significance of entry routes as point and non-point sources of pesticides in small streams”. Water Res. 36:835-842.

REPCAR. 2012. Residuos de plaguicidas agrícolas en aguas costeras del Caribe. Colombia, Costa Rica y Nicaragua. 2008-2011. En Internet: http://cep.unep.org/repcar/monitoreo-costero.

Rueda, L; Botelle, AV; Díaz G. 1997. "Presencia de plaguicidas organoclorados en dos sistemas lagunares del estado de Chiapas, México”. Rev. Int. Cont. Ambient. 13 (2), 55-61.

Tejeda, AW; Varca, LM; Calumpang, SMF. 1995. "Pesticides residues in food affected by crop protection practices”. ASEAN Food Journal. 10(3):94-98.

Umañas, GE. (2002). Manejo de Cuencas Hidrográficas y Protección de Fuentes de Agua. Universidad Nacional Agraria. Nicaragua. 27 pp.

Umañas, GE. (2002). Manejo de Cuencas Hidrográficas y Protección de Fuentes de Agua. Universidad Nacional Agraria. Nicaragua. 27 pp.

Varca, LM. 2012. “Pesticide residues in surface waters of Pagsanjan-Lumban catchment of Laguna Bay, Philippines”. Agricultural Water Management. 106:35-41.

Vryzas, Z; Vassiliou, G; Alexoudis, C; Papadopoulou, ME. 2009. "Spatial and temporal distribution of pesticides residues in surface water in notheastern Greece”. Water Research 43:1-10.

Wttmer, IK; Bader, HP; Scheidegger, R; Singer, H; Lück, A; Hanke, I; Carlsson, C; Stamm, C. 2010. “Significance of urban and agricultural land use for biocide and pesticide dynamics in surface waters”. Water Res. 44(9):2850-2862. 


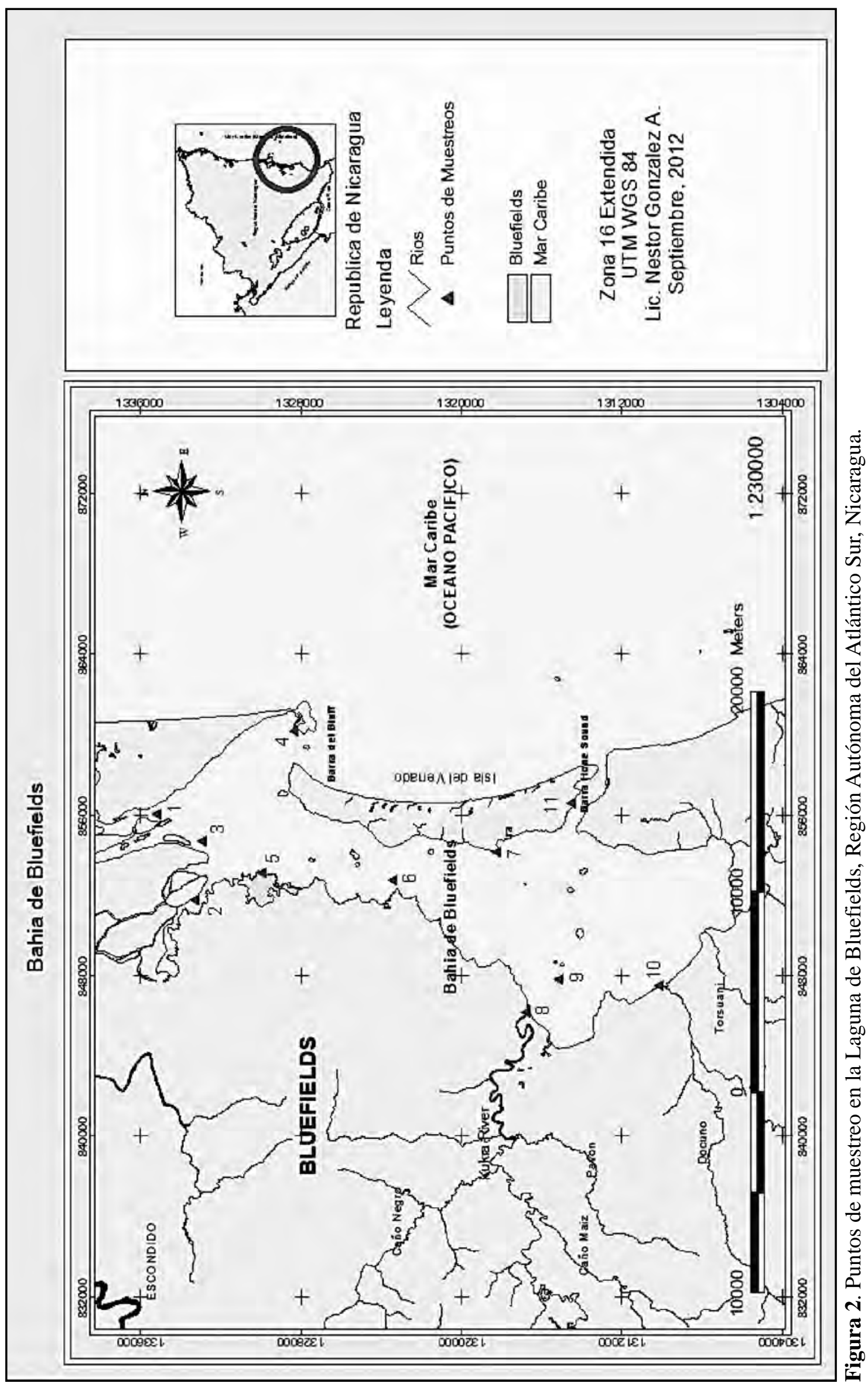

